\title{
Completeness Check of Experimental Fission Product Yield Data in EXFOR Database
}

\author{
Shin Okumura ${ }^{1, *}$ \\ ${ }^{1}$ NAPC-Nuclear Data Section, International Atomic Energy Agency
}

\begin{abstract}
The IAEA conducted the completeness assessment of fission product yield (FPY) data in EXFOR database against two bibliographies that were used in ENDF/B-VI and UKFY3.0 library evaluations. We found that 194 articles are found to be relevant for new entry.
\end{abstract}

\section{Introduction}

Since the discovery of nuclear fission, the fission product yield (FPY) is of interest from the fundamental understandings of nuclear physics as well as many nuclear energy applications. The nuclear fission is a complex phenomenon, whereby the nucleus forms compound, distort the shape, splits into two smaller fragments and release a large amount of energy with large amount of energy release. Until now, a complete picture of nuclear fission is not well understanded by any theories. It owing to the fact that the difficulty of sample preparation and experimental handling of heavy actinides.

The evaluation of major nuclear data libraries has been done mostly in 1990s. They generally cover fission yield data from the neutron-induced and spontaneous fission of 20-30 fissile materials including ones without sufficient experimental data. Therefore, such evaluation has been accomplished with both a guidance of available experimental data and utilizing empirical models $[1,2]$ to have consistencies in other fission observable such as prompt fission neutron multiplicity.

From 1990s, the IAEA Nuclear Data Section (NDS) has widely reviewed the user needs in these application fields in the previous IAEA Coordinated Research Projects (CRPs), e.g., "Compilation and evaluation of fission yield nuclear data" (1991-1996) [3], "Fission Product Yield Data for the Transmutation of Minor Actinide Nuclear Waste" (1997- 2002) [4], and the Technical Meeting on "Fission Product Yields: current status and perspectives" (2016) [5] to focus on the current situations of the experimental, modeling, and theoretical points of view for the FPY data evaluation. The recent increase in demand of the FPY data in various fields has triggered activities of developing new methods, codes and models for FPY evaluation. The IAEA-NDS will launch a nre CRP in 2020 for updating the evaluated FPY data for the neutron-induced fission of some important actinides.

The reliability of the evaluated data depends on the quality and authenticity of experimental data used in evaluation, where a complete set of the experimental FPY data is crucial. However, the experimental data stored in the international nuclear reaction database, also known as EXFOR [6], does not entirely cover all available FPY data. In this report, we

\footnotetext{
*e-mail: s.okumura@iaea.org
} 
outlines the procedures of completeness check of FPY data in the EXFOR database by comparing with the bibliographical references cited in the summary reports of the ENDF-B/VI [7] and UKFY3.0 [8] evaluations.

\section{Completeness assessment}

Since the procedure of completeness assessment of FPY data in EXFOR database comparing with FPY data used in ENDF-B/VI [7] and UKFY3.0 [8] evaluation summaries has been describe elsewhere [9-12], a brief description is given here.

The bibliographies of the ENDF-B/VI [7] and UKFY3.0 [8] evaluation summaries are converted into Author and Reference format that defined in the EXFOR format manual [13] (e.g., J, PR, 60, 534, 1941 for Phys. Rev. 60(1941)534). Two lists are merged and stored into the SQL database. Reference data are double-checked manually followed by a brief check using Python script, then Reference data are used in search of records EXFOR and CINDA. In case of a particular Reference has not found neither in EXFOR nor in CINDA, we identify the article using Crossref database for journal articles, online libraries, search engines, or electronic repositories such as International Nuclear Information System (INIS). If the electronic format is not available, hard copy of the article are collected through the IAEA Library.

The ENDF/B-VI evaluation summary [7] cites 1,602 articles in total, whose 924 articles have used to the actual evaluations while the rest of 678 articles have not used. The UKFY3.0 [8] evaluations cites 645 articles. Table 1 shows the number of articles categorised during our completeness assessment. Approximately 23\% of articles are overlapped in two bibliographies. After exclusion of the overlapped articles, the number of articles found to to be newly compiled are 194, while the articles that need to be checked with existing EXFOR entries are 54. The compilation has been started from April 2019 by NRDC data centres.

Figure 1 shows the number of FPY entries in EXFOR, which reflects the number of publications in a year. The first FPY measurements in EXFOR database was published in 1943. Until 1960s, FPY measurements are mostly dominated by the neutron induced fission of important actinides as a targets, namely Th, U, Pu, and Cf isotopes. The experimental FPY data drastically increase in 1970s. After 1980s, variations of target isotopes, e.g. Np, Am or $\mathrm{Cm}$, have increased while the majority of trend remains. Up to now, the majority of reactions are the neutron induced fission followed by the the spontaneous fission, photo-nuclear, and proton induced fission. By comparing number of FPY data in Fig. 1 extracted from EXFOR in July 2019 and December 2019, the number of entries increase in wide range of published year by efforts of NRDC data centres.

Table 1. The number of FPY references categorised during our assessment for those cited in evaluation summaries of ENDF/B-VI [7] and UKFY3.0 [8]. See Refs. [9-12] for details.

\begin{tabular}{lccccc}
\hline Source & Exist $^{\mathrm{a}}$ & Create new entry $^{\mathrm{b}}$ & Check $^{\mathrm{c}}$ & Not relevant $^{\mathrm{d}}$ & Total \\
\hline ENDF/B-VI & 618 & 154 & 36 & 116 & 924 \\
UKFY3.0 & 409 & 139 & 32 & 64 & 645 \\
\hline Total $^{\mathrm{e}}$ & 720 & 194 & 54 & 167 & 1203 \\
\hline
\end{tabular}

a Already compiled in EXFOR.

${ }^{b}$ Not found in EXFOR and need to create new entry.

${ }^{c}$ Need to check if all relevant data are compiled in EXFOR entry.

${ }^{\mathrm{d}}$ Not FPY or not relevant for EXFOR or the original article cannot identify.

e Total after elimination of overlap. 


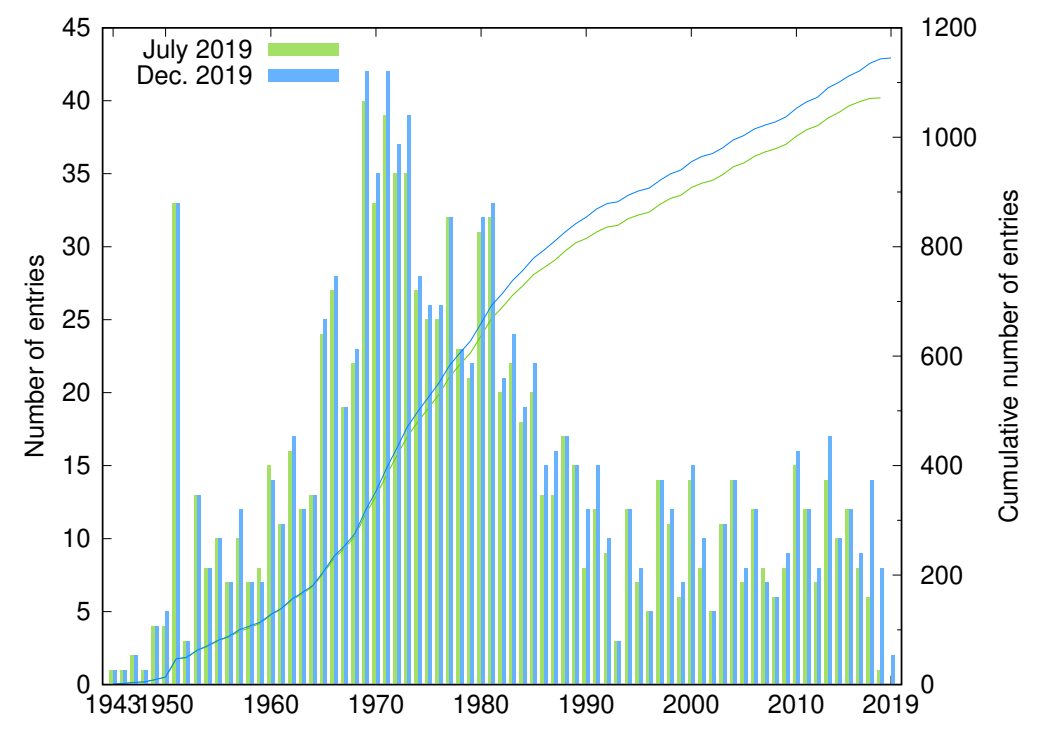

Figure 1. Number of FPY data entries in EXFOR based on the published year.

\section{Summary}

We reviewed the completeness of the FPY data in the EXFOR database by crosschecking against the bibliographies of the ENDF-B/VI and UKFY3.0 evaluation summaries. It was found that 194 references are relevant to new compilation of EXFOR entry, and 54 references must be checked with the existing EXFOR entries to ensure that the all relevant FPY data are properly compiled. The compilation has been started by data centres and the number of entries clearly increased after a half year from it has started.

\section{References}

[1] A.C. Wahl, Tech. Rep. LA-13928, Los Alamos National Laboratory (2002)

[2] D.G. Madland, T.R. England, Tech. Rep. LA-6595-MS, Los Alamos National Laboratory (1994)

[3] IAEA, Tech. Rep. IAEA-TECDOC-1168, IAEA (2000)

[4] IAEA, Tech. Rep. STI/PUB/1286, IAEA (2008)

[5] P. Dimitriou, F.J. Hambsch, S. Pomp (eds.), Tech. Rep. INDC(NDS)-0713, IAEA (2016)

[6] Experimental Nuclear Reaction Data (EXFOR), https://www-nds.iaea.org/exfor/

[7] T.R. England, B. Rider, Tech. Rep. ENDF-349, LA-UR-94-3106, Los Alamos National Laboratory (1994)

[8] R. Mills, Ph.D. thesis, University of Birmingham (1995)

[9] S. Okumura, NRDC Meeting 2019 Working paper (2019)

[10] S. Okumura, NRDC Memo CP-D/979 (2019)

[11] M. Fleming, T. Kawano, N. Otuka (eds.), Tech. Rep. INDC(NDS)-793, International Atomic Energy Agency (2019)

[12] T. Fukuda, S. Okumura, N. Otuka, submitted for EPJ Web of Conferences (2020)

[13] O. Schwerer (ed.), Tech. Rep. IAEA-NDS-207 Rev.2015/08, IAEA (2015) 